
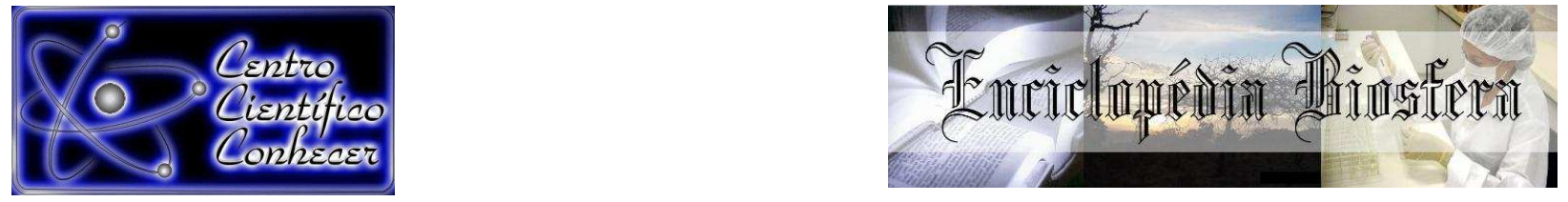

\title{
CARACTERÍSTICAS BIOMÉTRICAS E MASSA DE FRUTOS E SEMENTES DE Lecythis pisonis Cambess
}

Noemi Vianna Martins Leão ${ }^{1}$; Elizane Alves Arraes Araújoº ${ }^{2}$ Elizabeth Santos Cordeiro Shimizü; Sérgio Heitor Sousa Felipe ${ }^{4}$

${ }^{1}$ Pesquisadora do Laboratório de Sementes Florestais, Embrapa Amazônia Oriental, Caixa Postal 48, CEP 66095-100 - Belém, PA, Brasil. noemi.leao@embrapa.br

${ }^{2}$ Discente do curso de Engenharia Florestal da Universidade Federal Rural da Amazônia, CEP 66.077-830 - Belém, PA, Brasil.

${ }^{3}$ Analista do Laboratório de Sementes Florestais, Embrapa Amazônia Oriental, Caixa Postal 48, CEP 66095-100 - Belém, PA, Brasil.

${ }^{4}$ Pós graduando em Botânica, Laboratório de Cultura de Tecidos/BIOAGRO, Universidade Federal de Viçosa, CEP 36570-000 - Viçosa, MG, Brasil.

Recebido em: 03/10/2016 - Aprovado em: 21/11/2016 - Publicado em: 05/12/2016 DOI: 10.18677/EnciBio_2016B_015

\begin{abstract}
RESUMO
A castanha-de-sapucaia é uma espécie com altura média de $30 \mathrm{~m}$, natural da região amazônica, com aproveitamento desde a sua casca até as sementes. O objetivo do trabalho foi caracterizar biometricamente os frutos e sementes de duas matrizes de Lecythis pisonis Cambess. Os frutos foram coletados no município de Mãe do Rio (PA). Posteriormente, foram analisados 23 frutos e 100 sementes de cada árvore matriz. Para biometria dos frutos utilizou-se uma suta graduada, mensurando o comprimento e diâmetro, e, a massa fresca dos frutos obtida por meio de balança. Para o número de sementes por fruto, contabilizou-se o número total de sementes contidas em cada fruto. Para a biometria das sementes utilizou-se um paquímetro digital, mensurando comprimento, largura, espessura, e, a massa das sementes foi verificada com e sem arilo por meio de balança. Para os frutos e sementes analisadas, observou-se diferenças significativas para todas variáveis analisadas, exceto espessura das sementes, com os maiores valores presentes na matriz 1 . Os frutos da matriz 1 apresentaram média de 12,17 cm; 13,80 cm; 1,09 kg e 21,09 para as variáveis comprimento, diâmetro, massa fresca e número de sementes/ fruto, respectivamente, e, para as sementes, a média da matriz 1 foi $40,84 \mathrm{~mm} ; 24,06 \mathrm{~mm}$; $19,67 \mathrm{~mm} ; 11,74 \mathrm{~g}$ e $7,75 \mathrm{~g}$ para comprimento, largura, espessura, massa das sementes com arilo e sem arilo, respectivamente. Os frutos e sementes de castanha-sapucaia apresentam elevada variação para os parâmetros analisados, evidenciando elevado potencial para coletas de sementes.
\end{abstract}

PALAVRAS-CHAVE: Lecythidaceae, mensuração, variabilidade 


\title{
BIOMETRIC CHARACTERISTICS AND MASS OF FRUITS AND SEEDS Lecythis pisonis Cambess
}

\begin{abstract}
The sapucaia-nut is a species with an average height of 30 meters, native of the Amazon region, with use from its shell until the seeds. The objective of this study was to characterize biometrically the fruits and seeds of two matrices Lecythis pisonis Cambess. The fruits were collected in the town of Mãe do Rio (PA). 23 fruits and 100 seeds of two matrices were analyzed. Biometrics was carried out for fruits, measuring length and diameter, in centimeters, using graduated calipers. The mass was measured in kilograms and obtained the number of seeds per fruits. A caliper was used for seed biometrics, measuring length, width, thickness in millimeters and the mass of the seeds was measured with and without aryl and expressed in grams. For fruits, we observed significant differences for all variables, especially matrix 1 . For seeds, the matrix 1 was also higher than the matrix 2 , and all variables were significantly different, except for the variable thickness. The sapucaia-nut seeds and fruits have biometric differences between the matrices analyzed, which can infer the presence of morphometric variability between them.
\end{abstract}

KEYWORDS: Lecythidaceae, measurement, variability

\section{INTRODUÇÃO}

Pesquisas com frutos e sementes de espécies florestais nativas analisam, principalmente, os fatores fisiológicos, em detrimento aos aspectos biofísicos, como a biometria. Contudo, embora muitas pesquisas não deem relevância para os aspectos morfométricos de frutos e sementes de espécies nativas, esses estudos podem evidenciar a presença de variabilidade entre diferentes lotes de sementes, necessária para atender os projetos de recuperação e/ou recomposição de Áreas de Preservação Permanente e Reserva Legal. Neste contexto, faz-se necessário o desenvolvimento de pesquisas com espécies florestais nativas pouco estudadas, como é o caso de Lecythis pisonis Cambess.

A espécie $L$. pisonis ocorre na região Amazônica, nos estados do Acre, Amazonas, Pará e Rondônia, podendo ser encontrada em terra firme e várzea. É popularmente conhecida como castanha-de-sapucaia e sua altura varia de 20 a 30 $\mathrm{m}$ com tronco de 50 a $90 \mathrm{~cm}$ de diâmetro, sendo facilmente identificada na floresta pelo grande porte e caule estriado (SOUZA et al, 2014). Possui flores violáceas, vistosas e odoríferas (GUIMARÃES et al., 1993) que atrai os polinizadores.

Sua madeira é moderadamente pesada e muito utilizada como estacas, moirões dentre outras finalidades, possui fruto lenhoso e pode ser utilizado como adorno e como recipiente (LORENZI, 2002), em seu interior contem castanhas comestíveis sendo também muito apreciadas pelos animais, especialmente por morcegos que dispersam as sementes (MORI; PRANCE, 1990). Estudos realizados por CARVALHO et al. (2012) para a caracterização química da castanha-desapucaia, mostraram que a mesma apresenta altos teores de lipídios e proteínas, e podem ser indicadas como fonte de energia e proteínas em dietas. Os mesmos autores observaram elevados valores quanto ao conteúdo de ácidos graxos monoinsaturados, indicando um perfil lipídico benéfico ao sistema cardiovascular. 
Os aspectos morfométricos de frutos e sementes subsidiam informações para auxiliar na conservação florestal e indicar a presença de variabilidade entre indivíduos, bem como, a relação entre a diversidade fenotípica e o ambiente (FONTENELE et al., 2007). Além disso, pode fornecer informações para diferenciar espécies do mesmo gênero (ALVES et al., 2007) e para programas de melhoramento (GONÇALVES et al., 2013).

Considerando a necessidade de maiores estudos sobre os aspectos morfométricos de frutos e sementes de Lecythis pisonis, o presente estudo tem por objetivo realizar a caracterização biométrica de frutos e sementes dessa espécie, a fim de fornecer subsídios sobre a variabilidade da espécie e potencial seleção de árvores matrizes para coleta de sementes.

\section{MATERIAL E MÉTODOS}

O estudo foi desenvolvido no Laboratório de Sementes Florestais (LASF) da Embrapa Amazônia Oriental, em Belém, PA. Os frutos de L. pisonis foram coletados em duas árvores matrizes no mês de julho de 2015, no município de Mãe do Rio, mesorregião do Nordeste Paraense. As condições climáticas do município enquadram-se no tipo climático Am da classificação de Köppen, com precipitação média anual de $2.248 \mathrm{~mm}$ e temperatura média anual de $26,5^{\circ} \mathrm{C}$.

Após a coleta, os frutos foram encaminhados para o LASF, onde foram selecionados aleatoriamente 23 frutos de cada árvore matriz (matriz 01 e 02), observando-se as seguintes características fenotípicas: sadios, inteiros e sem deformações. A biometria dos frutos foi determinada com auxilio de uma suta graduada (Nakamura-30 cm) medindo-se o comprimento, considerando a região de inserção do pedúnculo até a região oposta ao mesmo, e, para o diâmetro, considerou-se a região intermediária do fruto. Para a massa fresca dos frutos utilizou-se uma balança eletrônica (Bioscale), com precisão de $0,1 \mathrm{~g}$.

Para o estudo das características biométricas das sementes foram selecionadas 100 sementes, aleatoriamente. As medições foram realizadas com auxílio de paquímetro digital (Mitutoyo-150 mm), com 0,01 mm de precisão. $O$ comprimento das sementes foi medido da região do hilo até a região oposta ao mesmo, sendo a espessura e largura, medidas na região intermediária. Para a massa fresca das sementes com e sem arilo foi utilizada uma balança de precisão (Sartorius, modelo BP 210S), com precisão de 0,0001 g. Na figura 1, encontra-se ilustrado o fruto de castanha-de-sapucaia onde é possível observar a estrutura do mesmo, assim como as sementes com e sem o arilo. 


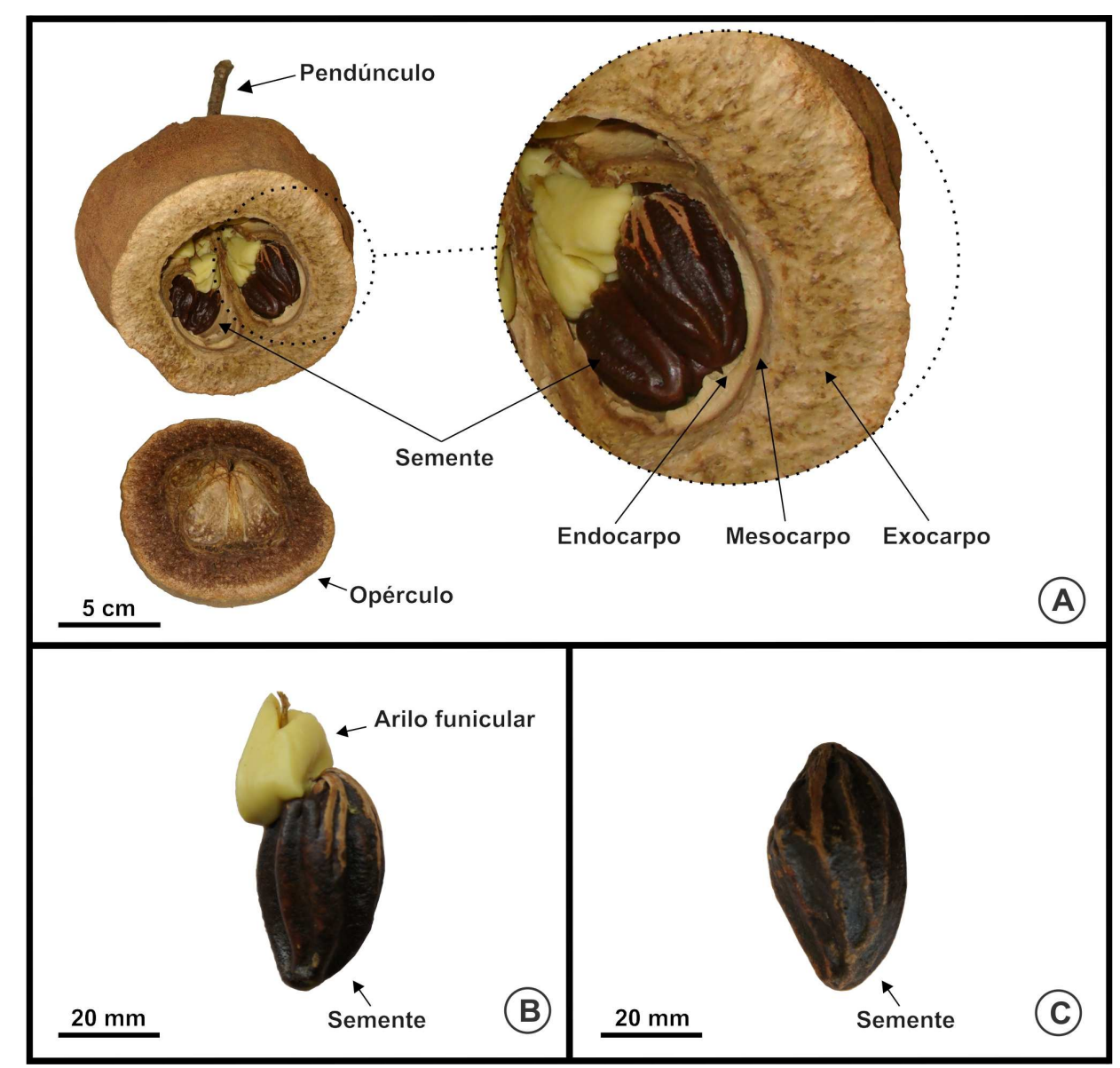

FIGURA 1. Ilustração do fruto $(A)$, semente com arilo funicular $(B)$, e semente sem arilo funicular $(\mathrm{C})$ da espécie Lecythis pisonis Cambess ocorrente no município de Mãe do Rio, PA. Fonte: Noemi V. M. Leão (A) e Elizabeth S. C. Shimizu (B) e (C).

Os parâmetros avaliados foram submetidos à análise descritiva, e, posterior análise de variância (ANOVA), para verificar se existiam diferenças entre os parâmetros das duas árvores matrizes, através do software SISVAR, ao nível de $5 \%$ de probabilidade e quando ocorreu significância, foi realizado o teste de Tukey, ao nível de $5 \%(p<0,05)$.

\section{RESULTADOS E DISCUSSÃO}

A análise descritiva dos frutos de $L$. pisonis (Tabela 1 ) evidenciou que os valores biométricos, de massa fresca e número de sementes por frutos apresentaram variações para ambas as matrizes analisadas. Em relação à assimetria, os valores observados na matriz 01 estão mais próximos de zero indicando maior aproximação da distribuição normal, quando comparado com a matriz 02. Os valores de coeficiente de variação foram maiores para os parâmetros de massa fresca e número de sementes por frutos para as duas matrizes analisadas, com valores acima de $40 \%$ na matriz 02.

Para a análise descritiva das sementes de $L$. pisonis (Tabela 1) as características biométricas e de massa fresca de sementes com e sem arilo apresentaram grande variação nas duas matrizes analisadas, evidenciando que pode existir elevada amplitude de tamanhos de sementes para essa espécie em 
povoamentos naturais. Os valores de assimetria evidenciaram que todas as variáveis analisadas das sementes estão próximas da distribuição normal. Para o coeficiente de variação, a espessura, massa fresca de sementes com e sem arilo para as duas matrizes apresentaram maiores valores.

Estudos realizados por BRAGA et al. (2007) evidenciaram tamanho médio das sementes igual a 43,1; 25,8 e 20,4 cm para comprimento, largura e espessura de sementes de $L$. pisonis, respectivamente. Esses valores estão próximos ao observado no presente estudo para a matriz 01, mas diferem dos resultados da matriz 02 que são inferiores. As variações biométricas verificadas nesse estudo para as duas árvores matrizes podem ser explicadas pelas interações existentes entre as características genéticas de cada individuo com as condições edafoclimáticas locais. Para as plantas não domesticadas é comum encontrar variações nas características biométricas (SANGALLI et al., 2012).

Segundo BRAGA et al. (2007), as sementes de L. pisonis apresentam elevada variação para comprimento, largura, espessura e massa das sementes, corroborando com os resultados do presente estudo. As condições genéticas das árvores matrizes e a interação dessas árvores com diferentes condições edáficas e microclimas podem propiciar elevados coeficientes de variação para as características fenotípicas das matrizes em populações naturais (LEÃO et al., 2015).

TABELA 1. Parâmetros biométricos e massa de frutos e sementes de Lecythis pisonis Cambess ocorrente no município de Mãe do Rio, PA. $\mathrm{N}=23$ para frutos e $\mathrm{N}=100$ para sementes.

\begin{tabular}{|c|c|c|c|c|c|c|c|}
\hline & Parâmetro & $\operatorname{Min}^{1}$ & Med & Max & As & $\mathrm{DP}$ & CV (\%) \\
\hline \multicolumn{8}{|c|}{ Frutos } \\
\hline \multirow{4}{*}{ 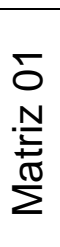 } & Comprimento (cm) & 9,90 & 12,17 & 13,90 & $-0,57$ & 1,13 & 9,24 \\
\hline & Diâmetro (cm) & 10,40 & 13,80 & 16,50 & $-0,43$ & 1,54 & 11,13 \\
\hline & Massa fresca $(\mathrm{kg})$ & 0,59 & 1,09 & 1,67 & 0,02 & 0,29 & 26,61 \\
\hline & $\begin{array}{l}\text { Número de sementes/ } \\
\text { fruto }\end{array}$ & 10,00 & 21,09 & 33,00 & 0,03 & 5,80 & 27,50 \\
\hline \multirow{4}{*}{ 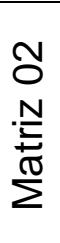 } & Comprimento $(\mathrm{cm})$ & 8,70 & 10,53 & 14,60 & 1,48 & 1,53 & 14,51 \\
\hline & Diâmetro (cm) & 9,70 & 12,74 & 18,60 & 1,46 & 1,83 & 14,39 \\
\hline & Massa fresca $(\mathrm{kg})$ & 0,46 & 0,83 & 1,95 & 1,90 & 0,35 & 41,87 \\
\hline & $\begin{array}{l}\text { Número de sementes/ } \\
\text { fruto }\end{array}$ & 5,00 & 10,52 & 27,00 & 1,74 & 5,19 & 49,29 \\
\hline \multicolumn{8}{|c|}{ Sementes } \\
\hline \multirow{5}{*}{ 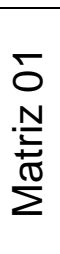 } & Comprimento (mm) & 28,98 & 40,84 & 48,85 & $-0,42$ & 3,94 & 9,64 \\
\hline & Largura $(\mathrm{mm})$ & 16,51 & 24,06 & 33,02 & 0,74 & 2,19 & 9,09 \\
\hline & Espessura (mm) & 11,56 & 19,67 & 28,26 & 0,26 & 2,29 & 11,64 \\
\hline & Massa com arilo (g) & 5,34 & 11,74 & 14,78 & $-0,90$ & 1,92 & 16,31 \\
\hline & Massa sem arilo (g) & 1,81 & 7,75 & 10,43 & $-1,08$ & 1,76 & 22,77 \\
\hline \multirow{5}{*}{ 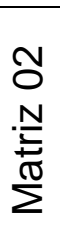 } & Comprimento (mm) & 22,79 & 34,70 & 42,91 & $-0,39$ & 4,20 & 12,10 \\
\hline & Largura (mm) & 12,85 & 22,22 & 28,88 & $-0,51$ & 2,84 & 12,80 \\
\hline & Espessura (mm) & 2,95 & 19,36 & 29,78 & $-0,91$ & 3,84 & 19,84 \\
\hline & Massa com arilo $(\mathrm{g})$ & 2,29 & 8,98 & 14,39 & $-0,54$ & 1,92 & 21,37 \\
\hline & Massa sem arilo $(\mathrm{g})$ & 2,36 & 6,16 & 10,43 & $-0,31$ & 1,59 & 25,73 \\
\hline
\end{tabular}

Min: mínimo valor; Med: média; Max: máximo valor; As: assimetria; DP: desvio padrão e CV: coeficiente de variação. Fonte: Elaborado pelos autores 
Para verificar se há diferenças entre as características biométricas e massa de frutos e sementes de $L$. pisonis das duas árvores matrizes, realizou-se a análise de variância e comparação de médias. Os resultados evidenciaram diferenças significativas para todos os parâmetros analisados dos frutos e sementes $(p \leq 0,05)$, com exceção da espessura das sementes $(p \geq 0,05)$ (Figura 2 e 3 ).

Os frutos e sementes da matriz 01 apresentaram maiores médias em relação à matriz 02 , indicando a presença de variabilidade morfométrica entre as matrizes. Esses resultados divergem dos encontrados por SANTOS et al. (2013) para Aspidosperma desmanthum, onde os autores analisaram sete árvores matrizes e não observaram predomínio de nenhuma árvore matriz sobre as demais em todos os aspectos biométricos de frutos e sementes analisados.

Em relação à variação fenotípica de frutos e/ou sementes de espécies florestais, pesquisas tem evidenciado presença de variabilidade, como em, Dipteryx alata (ZUFFO et al., 2014), Schizolobium parahyba (FREIRE et al., 2015) e Bertholletia excelsa (TEIXEIRA et al., 2015), corroborando com a presente pesquisa. A presença de variabilidade genética em sementes florestais é fundamental para manter a sustentabilidade das comunidades vegetais recuperadas (SOUZA et al., 2013).
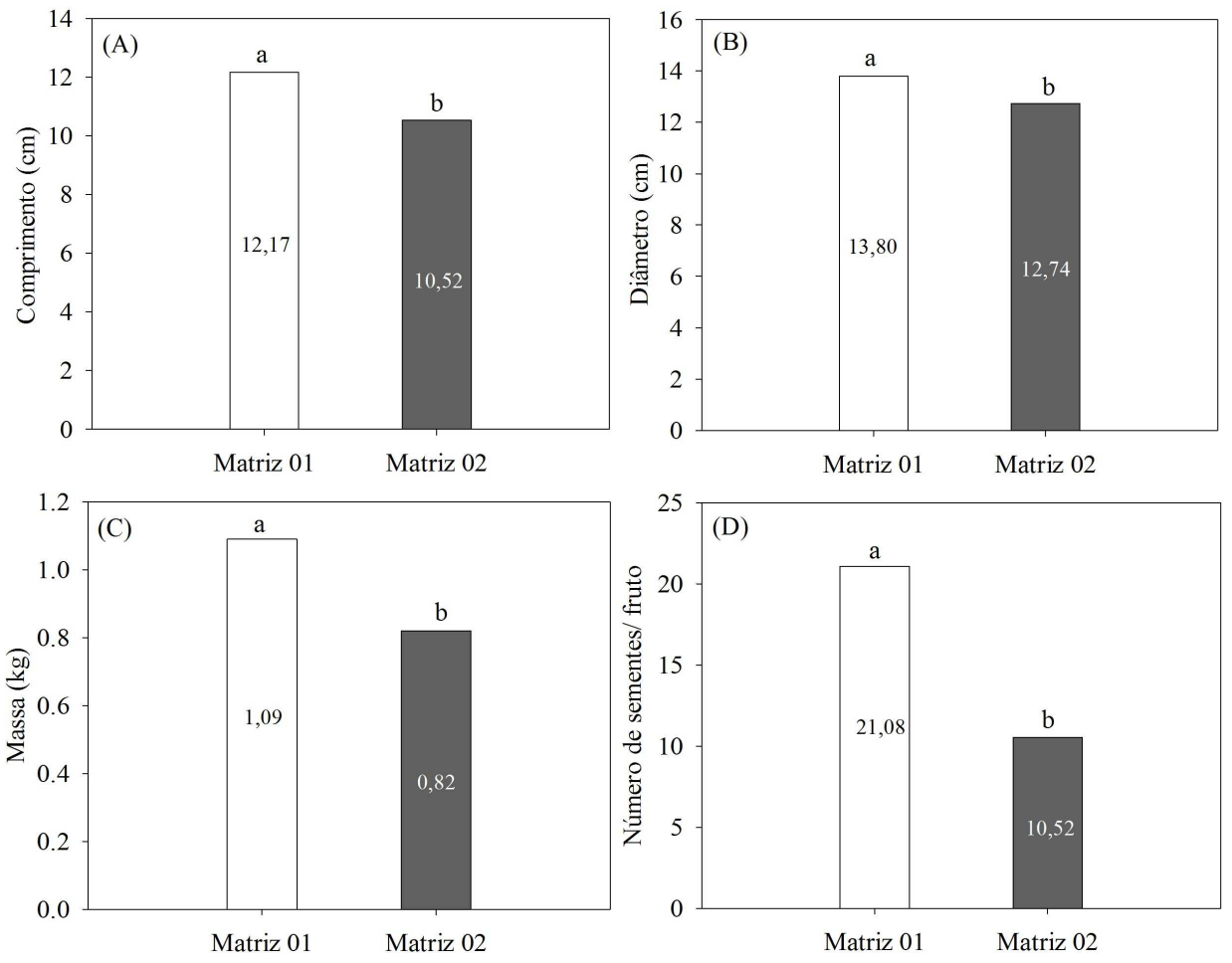

FIGURA 2. Médias de comprimento (A), diâmetro (B), massa $(C)$ e número de sementes (D) dos frutos de duas matrizes de Lecythis pisonis Cambess ocorrente no município de Mãe do Rio, PA. Fonte: Elaborado pelos autores 

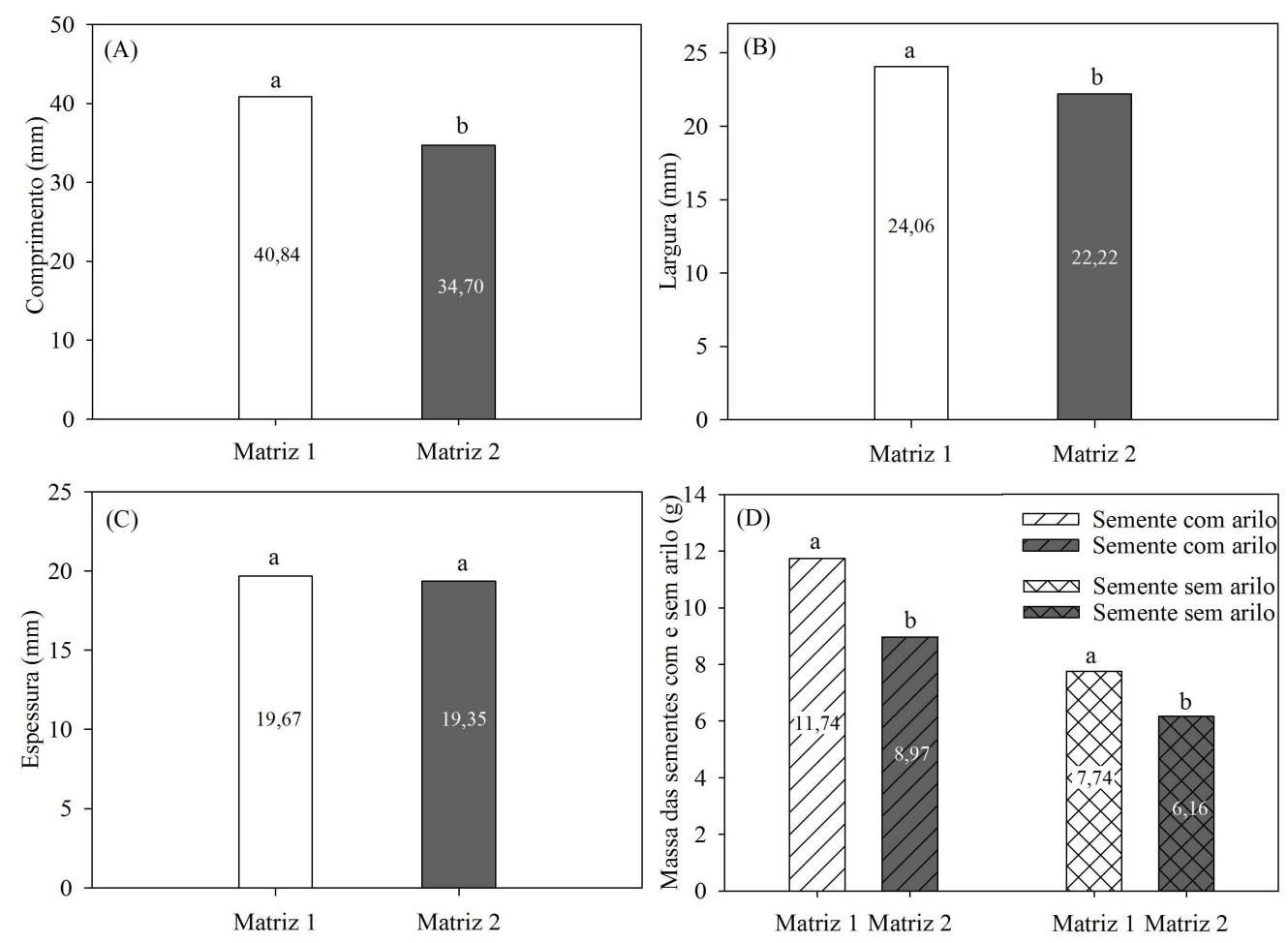

FIGURA 3. Médias de comprimento (A), largura (B), espessura (C) e massa de sementes com e sem arilo (D) de sementes de duas matrizes de Lecythis pisonis Cambess ocorrente no município de Mãe do Rio, PA. Fonte: Elaborado pelos autores

\section{CONCLUSÕES}

Os frutos e sementes de castanha-de-sapucaia apresentaram elevada variação para os parâmetros analisados, bem como, diferenças para as características biométricas e massa de frutos e sementes entre as matrizes.

As matrizes 01 e 02 apresentaram elevado potencial para coletas de sementes destinadas a recuperação e/ou recomposição de Áreas de Preservação Permanente e Reserva Legal.

\section{AGRADECIMENTOS}

Aos assistentes e técnicos do Laboratório de Sementes Florestais, da Embrapa Amazônia Oriental pelo apoio no desenvolvimento do trabalho e a Fundação Amazônia de Amparo a Estudos e Pesquisas (FAPESPA) pela disponibilização do recurso.

\section{REFERÊNCIAS}

ALVES, E. U.; BRUNO ALCÂNTARA, R. L.; ALVES, A. U.; ALVES, A. U.; CARDOSO, E. A.; GALINDO, E. A.; JÚNIOR, J. M. B. Germinação e biometria de frutos e sementes de Bauhinia divaricata L. (Leguminosae). Sitientibus Série Ciências Biológicas, v. 7, n. 3, p. 193-198, 2007. Disponível em: http://www.scielo.br/scielo.php?script=sci_nlinks\&ref=000101\&pid=S1516$0572201300020000100003 \& \operatorname{lng}=\mathrm{pt}$ 
BRAGA, L. F.; SOUSA, M. P.; GILBERT,S.; de CARVALHO, M. A. C. Caracterização morfométrica de sementes castanha de sapucaia (Lecythis pisonis Cambess Lecythidaceae). Revista de Ciências Agro-ambientais. v. 5, n.1, p. 111-116, 2007. Disponível em: http://www.unemat.br/revistas/rcaa/docs/vol5/11_artigo_v5.pdf

CARVALHO, I. M. de.; QUEIRÓS, L. D.; BRITO, L. F.; SANTOS, F. A.; BANDEIRA, A. V. M.; SOUZA, A. L. de; QUEIROZ, J. H. de; Caracterização Química da Castanha de Sapucaia (Lecythis pisonis Cambess.) da Região da Zona da Mata Mineira. Bioscience Journal, Uberlândia, v. 28, n. 6, p. 971-977, Nov./Dec. 2012. Disponível http://www.seer.ufu.br/index.php/biosciencejournal/article/viewFile/14063/11108

em:

FONTENELE, A. C. F.; ARAGÃO, W. M.; RANGEL, J. H. DE A. Biometria de Frutos e Sementes de Desmanthus virgatus (L) Willd Nativas de Sergipe. Revista Brasileira de Biociências, Porto Alegre, v. 5, suplemento 1, p. 252-254, jul. 2007. Disponível em: http://www.ufrgs.br/seerbio/ojs/index.php/rbb/article/viewFile/275/244

FREIRE, J. M.; PINA-RODRIGUES, F.C.M.; SANTOS, A.L.F.; PEREIRA, M.B. Intraand inter-population variation in seed size and dormancy in Schizolobium parahyba (Vell.) Blake in the Atlantic Forest. Ciência Florestal, v. 25, n. 4, p. 897-907, 2015.

GONÇALVES, L.G.V.; ANDRADE, F.R.; MARIMON JUNIOR, B.H.; SCHOSSLER, T.R.; LENZA, E.; MARIMON, B.S. Biometria de frutos e sementes de mangaba (Hancornia speciosa Gomes) em vegetação natural na região leste de Mato Grosso, Brasil. Revista de Ciências Agrárias, v.36, n.1, p.31-40, 2013. Disponível em: http://www.scielo.mec.pt/scielo.php?script=sci_arttext\&pid=S0871-

$018 X 2013000100006$

GUIMARÃES, E. F.; MAUTONE, L.; RIZZINI, C. T.; MATOS FILHO, A. Árvores do Jardim Botânico do Rio de Janeiro. Rio de Janeiro: Jardim Botânico, 1993. 234 p.

LEÃO, N.V.M.; FELIPE, S.H.S.; SHIMIZU, E.S.C.; DOS SANTOS FILHO, B.G.; KATO, O.R.; BENCHIMOL, R. L. Biometria e diversidade de temperaturas e substratos para a viabilidade de sementes de ipê amarelo. Informativo ABRATES, v. 25, n. 1, p. 50-54, 2015. Disponível em: http://www.abrates.org.br/images/Informativo/v25_n1/018_2014_Elizabeth_Shimizu.p df

LORENZI, H. Árvores Brasileiras: Manual de identificação e cultivo de plantas arbóreas nativas do Brasil. 4. ed. Nova Odessa: Plantarum, 2002, 368 p. v. 1.

MORI, S.A.; PRANCE, G. T. Lecythidaceae - Part II: The zygomorphic-flowered New World genera (Bertholletia, Corythophora, Couratari, Couroupita, Eschweilera, and Lecythis).Flora Neotropica Monographs, v. 21, n. 2, p. 1-376, 1990.

SANGALLI, A.; VIEIRA, M. C.; SCALON, S. P. Q.; ZÁRATE, N. A. H.; SILVA, C. B.; RIBEIRO, I. S. Morfometria de frutos e sementes e germinação de carobinha (Jacaranda decurrens subsp. symmetrifoliolata Farias \& Proença), após 0 
armazenamento. Revista Brasileira de Plantas Medicinais, v. 14, n. 2, p. 267-275, 2012. Disponível em: http://www.scielo.br/scielo.php?script=sci_arttext\&pid=S151605722012000200003> http://dx.doi.org/10.1590/S1516-05722012000200003

SANTOS, S.O.; GALLO, R.; RONDON NETO, R.M. Biometria de diásporos de peroba-mica (Aspidosperma desmanthum Muell. Arg.). ENCICLOPÉDIA BIOSFERA, Centro Científico Conhecer - Goiânia, v.9, n.17; 2013. Disponível em: http://www.conhecer.org.br/enciclop/2013b/CIENCIAS\%20AGRARIAS/biometria.pdf

SOUZA, A. S.; MARGALHO, L.; PRANCE, G. T.; GURGEL, E. S. C.; GOMES, J. I.; CARVALHO, L. T. de.; MARTINS-DA-SILVA, R. C. V.; Conhecendo Espécies de Plantas da Amazônia: Sapucaia (Lecythis pisonis Cambess. - Lecythidaceae). Embrapa Amazônia Oriental. Comunicado Técnico 250, 2014. Disponível em: https://www.infoteca.cnptia.embrapa.br/bitstream/doc/999043/1/COM250.pdf

SOUZA, D.C.L.; SILVA-MANN, R.; FERREIRA, R.A.; GOMES, L.J.; ALMEIDA, T.D.S.; OLIVEIRA, A.D.S.; PEREIRA, G.S.; GOIS, I.B. Produção de frutos e características morfofisiológicas de Schinus terebinthifolius Raddi., na região do baixo São Francisco, Brasil. Revista Árvore, v. 37, n. 5, p. 923-932, 2013. Disponível em: http://www.scielo.br/scielo.php?pid=S010067622013000500015\&script=sci_abstract\&tlng=pt

TEIXEIRA, R.A.; PEDROZO, C.Â.; DA COSTA, E.K.L.; BATISTA, K.D.; TONINI, H.; PESSONI, L.A. Correlações e divergência fenotípica entre genótipos cultivados de castanha-do-Brasil. Scientia Forestalis, Piracicaba, v. 43, n. 107, p. 523-531, set. 2015. Disponível em: http://www.ipef.br/publicacoes/scientia/nr107/cap03.pdf

ZUFFO, A.M.; ANDRADE, F.R.; JÚNIOR, J.M.Z. Caracterização biométrica de frutos e sementes de baru (Dipteryx alata Vog.) na região leste de Mato Grosso, Brasil. Revista de Ciências Agrárias, v. 37, n. 4, p. 463-471, 2014. Disponível em: http://www.scielo.mec.pt/scielo.php?script=sci_arttext\&pid=S0871-

018X2014000400012 